\title{
Efficacy of Qurmin on Post- Operative Lower Urinary Tract Symptoms After Photoselective Vaporization of the Prostate: A Multicentre Case-Control Study
}

\begin{abstract}
Keywords: Photoselective vaporization of the prostate; Benign Prostatic hyperplasia; Curcumin; Lower urinary tract symptoms

Abstract

Objective: To evaluate the efficacy of highly bioavailable curcumin complex on postoperative lower urinary symptoms in patients treated with photoselective vaporization of the prostate (PVP) for lower urinary tract symptoms and benign prostatic enlargement (LUTS/BPE) in a multicentre randomized controlled study.
\end{abstract}

Materials and Methods: A consecutive series of patients with LUTS-BPE undergoing PVP were enrolled. Patients were randomized in a 2:1 ratio to oral highly bioavailable curcumin complex (Qurmin ${ }^{\circledR}$, Naturneed, Italy), twice a day for 10 days then once daily for 20 days postoperatively or no treatment. Clinical and demographic characteristics of all patients were collected. Patients were evaluated preoperatively, on day 1 (after catheter removal), on day 15 and on day 30 using the International Prostate Symptom Score (IPSS), National Institute of Health Chronic Prostatitis Symptom Index (NIH-CPSI) and Pain Urgency Frequency (PUF) questionnaires to assess the efficacy of the treatment.

Results: Overall 106 patients were randomized to curcumin complex treatment (71/106:67\%) or no treatment (35/106:33\%). Preoperatively no significant differences in terms of age, PV, IPSS; Qmax, NIH-CPSI and PUF were recorded. Patients in both groups presented improvements in terms of IPSS, NIH-CPSI and PUF scores on day 15 and on day 30 when compared to day $1(p<0,01)$. On day 15 patients on active treatment group presented a better improvement in terms of urinary symptoms ( $\triangle 15-1$ IPSS score: $6,0 \pm 7,6$ vs $3,1 \pm 4,6$, $p=0,001)$ and pain $(\Delta 15-1$ PUF pain : $1,7 \pm 4,2$ vs $-0,8 \pm 4,1, p=0,001)$. As well on day 30 patients on active treatment group presented a better improvement in terms of urinary symptoms ( $\triangle 30$-1 IPSS score: 9,0 $\pm 7,6$ vs $6,2 \pm 6,4, p=0,001)$ and pain $(\Delta 30-1$ PUF pain: $3,3 \pm 5,3$ vs $1,6 \pm 4,5$, $\mathrm{p}=0,001$ ). No significant differences in terms of $\mathrm{NIH}-\mathrm{CPS}$ improvements were recorded between groups. No adverse events or side effects have been recorded in the treated group.

Conclusions: Patients treated with highly bioavailable curcumin complex after PVP reported a significantly better improvement in symptoms and pain when compared to no treatment. Further studies should confirm our results.

\section{Introduction}

GreenLight laserphotoselective vaporization of the prostate (PVP) is one of the most versatile and safe procedures to treat benign prostatic obstruction (BPO), with the possibility to perform different surgical procedure (standard or anatomical vaporization or pure enucleation (GreenLEP)) [1-3]. Some of the advantages of PVP are shorter catheterization time and hospital stay when compared to trans-urethral resection of the prostate (TURP) [4]. Post-operative urinary retention leads to patients' discomfort, prolonged hospital stay and increasedhealth care costs [5].

\section{Journal of}

\section{Urology \& Nephrology}

Cindolo $L^{1}$, Lombardo $R^{2}$, Destefanis $P^{3}$, Varvello $F^{4}$, Oriti $R^{5}$, Tuccio $A^{5}$, Ruggera $L^{6}$, De Rienzo $\mathbf{G}^{7}$, Gentile $\mathbf{G}^{8}$, Ferrari $\mathrm{G}^{9}$ and De Nunzio $\mathrm{C}^{2}$

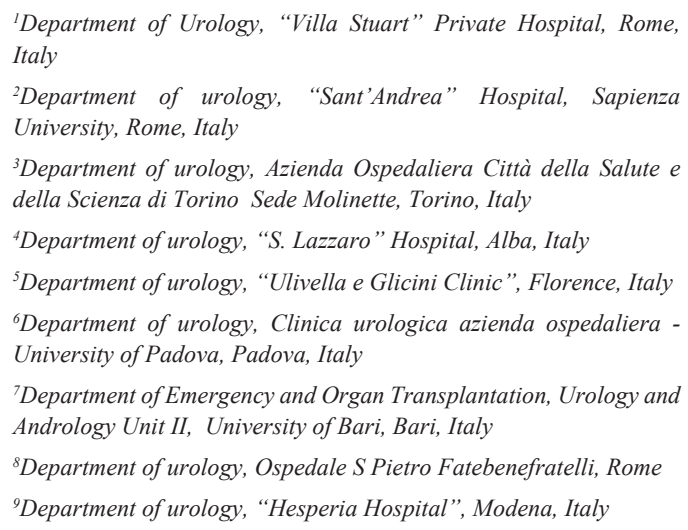

\section{${ }^{*}$ Address for Correspondence}

Lombardo R, Department of urology, "Sant' Andrea" Hospital, Sapienza University, Rome, Italy, Fax: 39-06-33775059, Tel: 39-06-33775306; Email: rlombardo@me.com

Submission: 12 August, 2021

Accepted: 15 September, 2021

Published: 20 September, 2021

Copyright: () 2021 Cindolo L, et al. This is an open access article distributed under the Creative Commons Attribution License, which permits unrestricted use, distribution, and reproduction in any medium, provided the original work is properly cited.

In the early post-operative period after TURP or PVP, patients may experience some discomfort. It is estimated that $5-10 \%$ of the patients may suffer from transient urinary incontinence, $5-20 \%$ of the patients may present transient dysuria and $10-50 \%$ of the patients may suffer from transient haematuria in the first post-operative month [5-6]. Regarding treatment of early postoperative LUTS very few studies have evaluated the role of possible medications to improve symptoms. The available evidence suggest that antimuscarinics or alpha blocker may improve post-operative LUTS however no definitive conclusion has been made yet [2]. Curcuminis an active compound contained in turmeric. In vitro studies have suggested curcuma acts attenuating the expression of IL1B, IL6 and TNF alfa resulting in an anti-inflammatory effect. Clinical studies have as well confirmed its role in reducing inflammation in patients with gastritis and ostheo-artritis. However, only a small study has evaluated the role of curcumin in patients with LUTS due to BPH while no study has evaluated the possible role of curcuminin the management of early post PVP symptoms [3-6].

With this knowledge in mind, aim of our study was to evaluate in a randomized clinical trial the efficacy of an oral highly bioavailable curcumin complexin improving postoperative dysuria in patients undergoing PVP. 
Citation: Cindolo L, Lombardo R, Destefanis P, Varvello F, Oriti R, et al. Efficacy of Qurmin on Post-Operative Lower Urinary Tract Symptoms After Photoselective Vaporization of the Prostate: A Multicentre Case-Control Study. J Urol Nephrol. 2021;8(1): 5.

\section{Materials and Methods}

After an internal review board approval, a consecutive series of patients with LUTS-BPE undergoing PVP were enrolled. All patients signed an informed consent, and all the procedures were performed in accordance with the Declaration of Helsinki. Any patient with prior history of prostatic or urethral surgery, urethral stricture, neuro-vesical dysfunction, and/or prostate cancer was excluded. As well patients with uncontrolled diabetes, obstruction of the bile duct, cholangitis, liver disease, gallstones and any other biliary disease were excluded.

\section{Preoperative Evaluation}

Clinical data including age, body mass index (BMI), haemoglobin levels, International Prostate Symptom Score (IPSS), QoL score, national institute of health chronic prostatitis symptom index (NIHCPSI), pain urgency frequency (PUF), prostate specific antigen (PSA), prostatic volume (PV) and post-void residual were retrospectively collected. Indications for surgery included a pre-operative IPSS $\geq 12$ points and/or a maximal urinary flow rate (Qmax) $<15 \mathrm{~mL} / \mathrm{s}$, and/ or a prostate volume $>40 \mathrm{gr}$ on transrectal ultrasound and/or nonresponder to LUTS medical treatment.

\section{Surgical Tecnique}

Anti-platelet and anticoagulant drugs were stopped at least 1 week before the operation. Considering the potential effects of curcumin on coagulation, patients under anti-platelet and anticoagulant drugs were carefully monitored during treatment (serum tests every week). In case of any coagulation disorder curcumin was stopped and patients were excluded from the study. A complete blood count, creatininemia, platelet count, bleeding profile, coagulation profile and urine culture were collected pre- and postoperatively. An enema was routinely administered the afternoon before surgery for bowel preparation. Elastic compressive stocking was used as mechanical prophylaxis for deep vein thrombosis and third-generation cephalosporin was administered intravenously when anesthesia was initiated and repeated 8 hours postoperatively as antibiotic prohylaxis. A photoselective vaporization of the prostate performed with a 26Ch continuous irrigation resectoscope. Saline solution was used as irrigation fluid and aspostoperative irrigation fluid. Bladder irrigation was maintained for at least 24 hours. The catheter was removed 1 hour after discontinuing bladder irrigation [7].

\section{Post-operative evaluation and randomization}

According to the CONSORT guidelines, simple randomization was made using the sealed envelope method. Patients were randomized in a 2:1 ratio to an oral highly bioavailable curcumin complex (Qurmin', Naturneed, Italy), twice a day for 10 days then once daily for 20 days postoperatively or no treatment. Patients were evaluatedpostoperatively on day 1 (after catheter removal), on day 15 and on day 30 using the International Prostate Symptom Score (IPSS), National Institute of Health Chronic Prostatitis Symptom Index (NIH-CPSI) and pain urgency frequency (PUF) questionnaires to assess the efficacy of Qurmin treatment.

\section{Statistical Analysis and Power calculation}

Statistical analysis was performed using the SPSS v. 22.0 software.
Evaluation of data distribution showed a non-normal distribu $\neg$ tion of the study data set. Differences between groups of patients in medians for quantita $\neg$ tive variables and differences in distributions for categorical variables were tested with the Kruskal-Wallis one-way analysis of variance and chi-square test, respectively. Wilcoxon rank test was used to evaluate improvements in questionnaires scores.

Power calculation: Sample size calculation was based on an initial hypothesis of 3 IPSS points difference in terms of improvement between groups. An improvement of $6 \pm 5$ points was hypothesized in the no treatment group. An alpha value of $5 \%$ and a power of $80 \%$ were used. Sample size needed to prove the hypothesis including a $20 \%$ dropout rate was 118 patients.

\section{Results}

Overall, 106 patients were randomized to Qurmin treatment (71/106:67\%) or no treatment (35/106:33\%). Preoperatively patients in the active treatment group were younger when compared to patients in the sham group ( 65 vs $70, p=0,001$ ). No significant differences in terms of PV, IPSS; Qmax, PSA, NIH-CPSI and PUF were recorded (Table 1)

On day 15 patients in the active treatment group presented statistically significant improvements in terms of IPSS (Day 1: 14; 10/22 vs Day 15: 9; 4/11; p=0,001), PUF (Day 1: 13; 10/17vs Day 15: 9; 6/13; $\mathrm{p}=0,001)$ and NIH-CPS $(16 ; 12 / 25$ vs $12 ; 7 / 17 ; \mathrm{p}=0,001)$ (Table 2). On Day 30 patients in the active treatment group presented statistically significant improvements in terms of IPSS (Day 1: 14; 10/22 vs Day 30: 5; 3/9; $\mathrm{p}=0,001$ ), PUF (Day $1: 13 ; 10 / 17$ vs Day 30 : 7; 4/10; $\mathrm{p}=0,001$ ) and NIH-CPS (Day 1: 16; $12 / 25$ vs Day 30 :7; 3/12; $\mathrm{p}=0,001)$ (Table 2).

When comparing both groups, on day 15 patients on active treatment group presented a better improvement in terms of urinary symptoms ( $\Delta 15-1$ IPSS score: $4: 1 / 10$ vs $3: 0 / 7, \mathrm{p}=0,001)$ and pain ( $\Delta 15-1$ NIH pain: $1: 0 / 4$ vs $0:-3 / 0 ; p=0,001)$ when compared to the no treatment arm. As well on day 30 patients on active treatment group presented a better improvement in terms of urinary symptoms $(\Delta 30$ 1 IPSS score: $9: 4 / 13$ vs $6: 2 / 11, p=0,001)$ and pain $(\Delta 30-1 \mathrm{NIH}$ pain: $3: 0 / 7$ vs $0: 0 / 3 ; \mathrm{p}=0,001$ )when compared to the no treatment arm. No significant differences in terms of NIH-CPS improvements were recorded between groups (Table 3).

Patients undergoing Qurmin treatment presented no adverse events related to drug treatment. Moreover, patients under anticoagulant/antiplatelet drugs did not present any coagulation abnormalities on serum tests during treatment.

Table 1: General Characteristics and according to treatment group

\begin{tabular}{|c|c|c|c|c|}
\hline & OVERALL & CONTROL & ACTIVE & p \\
\hline Age & $66(61 / 75)$ & $70(63 / 74)$ & $65(60 / 72)$ & 0.001 \\
\hline Prostate Volume & $59(40 / 71)$ & $55(40 / 70)$ & $60(47 / 75)$ & 0.461 \\
\hline Operative time & $60(47 / 65)$ & $60(50 / 60)$ & $60(40 / 70)$ & 0.851 \\
\hline PSA & $2.7(1.7 / 4.0)$ & $2.3(1.4 / 3.5)$ & $3.2(2.1 / 4.2)$ & 0.232 \\
\hline Qmax & $9(6 / 10)$ & $9(7 / 10)$ & $9(6 / 11)$ & 0.625 \\
\hline IPSS & $21(16 / 26)$ & $21(11 / 26)$ & $20(16 / 26)$ & 0.488 \\
\hline PUF & $13(8 / 18)$ & $16(8 / 18)$ & $17(9 / 17)$ & 0.402 \\
\hline NIH-CPS & $19(13 / 23)$ & $22(16 / 28)$ & $18(11 / 22)$ & 0.065 \\
\hline
\end{tabular}


Citation: Cindolo L, Lombardo R, Destefanis P, Varvello F, Oriti R, et al. Efficacy of Qurmin on Post-Operative Lower Urinary Tract Symptoms After Photoselective Vaporization of the Prostate: A Multicentre Case-Control Study. J Urol Nephrol. 2021;8(1): 5.

Tabe 2: Post-TURP symptomsaccording to treatment groupatday 1,15 and 30 .

\begin{tabular}{|c|c|c|c|}
\hline \multicolumn{4}{|c|}{ Control Group } \\
\hline & Day 1 & Day 15 & Day 30 \\
\hline IPSS & $12(8 / 16)$ & $9(4 / 13)$ & $4(3 / 7)$ \\
\hline IPSS voiding & $4(2 / 7)$ & $4(0 / 6)$ & $2(0 / 3)$ \\
\hline IPSS storage & $6(5 / 10)$ & $4(3 / 7)$ & $3(2 / 5)$ \\
\hline IPSS QoL & $3.5(2 / 4)$ & $2(2 / 3)$ & $1(0 / 2)$ \\
\hline PUF total & $11(9 / 15)$ & $8(4 / 11)$ & $7(4 / 10)$ \\
\hline PUF symptom & $8(6 / 9)$ & $5(3 / 9)$ & $3(2 / 7)$ \\
\hline PUF bother & $4(2 / 5)$ & $2(1 / 4)$ & $2(0 / 3)$ \\
\hline $\mathrm{NIH}$ total & $10(7 / 19)$ & $9(3 / 15)$ & $7(3 / 12)$ \\
\hline $\mathrm{NIH}$ pain & $0(0 / 4)$ & $3(0 / 5)$ & $3(0 / 6)$ \\
\hline NIH micturition & $3(2 / 6)$ & $3(1 / 4)$ & $2(0 / 3)$ \\
\hline NIH quality & $5(4 / 9)$ & $3(1 / 5)$ & $2(1 / 4)$ \\
\hline \multicolumn{4}{|c|}{ Active treatment Group } \\
\hline IPSS & $14(10 / 22)$ & $9(4 / 11)$ & $5(3 / 9)$ \\
\hline IPSS voiding & $6(2 / 11)$ & $2(0 / 7)$ & $1(0 / 3)$ \\
\hline IPSS storage & $9(6 / 12)$ & $6(3 / 8)$ & $3(2 / 5)$ \\
\hline IPSS QoL & $3(2 / 5)$ & $2(1 / 3)$ & $1(0 / 2)$ \\
\hline PUF total & $13(10 / 17)$ & $9(6 / 13)$ & $7(4 / 10)$ \\
\hline PUF symptom & $8(6 / 11)$ & $7(4 / 9)$ & $4(2 / 6)$ \\
\hline PUF bother & $4(3 / 6)$ & $3(2 / 4)$ & $2(1 / 4)$ \\
\hline $\mathrm{NIH}$ total & $16(12 / 25)$ & $12(7 / 17)$ & $7(3 / 12)$ \\
\hline NIH pain & $7(2 / 11)$ & $5(0 / 8)$ & $3(0 / 6)$ \\
\hline NIH micturition & $4(2 / 6)$ & $3(1 / 5)$ & $2(0 / 3)$ \\
\hline NIH quality & $6(4 / 9)$ & $4(1 / 7)$ & $2(1 / 4)$ \\
\hline
\end{tabular}

Table 3: Improvements in bothgroups in urinarysymptoms from baseline

\begin{tabular}{|c|c|c|c|c|c|c|}
\hline & \multicolumn{3}{|c|}{15 days- Baseline } & \multicolumn{3}{c|}{30 Days-Baseline } \\
\hline & Control & Active & p & Control & Active & p \\
\hline$\Delta$ IPSS & $3(0 / 7)$ & $4(1 / 10)$ & 0.001 & $6(2 / 11)$ & $9(4 / 13)$ & 0.001 \\
\hline$\Delta$ IPSS storage & $1(0 / 4)$ & $2(0 / 6)$ & 0.283 & $3(2 / 6)$ & $5(2 / 7)$ & 0.222 \\
\hline$\Delta$ PUF total & $3(0 / 7)$ & $3(1 / 7)$ & 0.535 & $5(1 / 9)$ & $6(3 / 9)$ & 0.335 \\
\hline$\Delta$ PUF symptom & $1(0 / 5)$ & $2(0 / 4)$ & 0.589 & $4(0 / 7)$ & $4(2 / 7)$ & 0.381 \\
\hline$\Delta$ PUF bother & $1(0 / 2)$ & $1(0 / 3)$ & 0.383 & $2(0 / 4)$ & $2(1 / 4)$ & 0.535 \\
\hline$\Delta$ NIH total & $3(0 / 7)$ & $3(1 / 7)$ & 0.535 & $6(4 / 11)$ & $5(1 / 8)$ & 0.121 \\
\hline$\Delta$ NIH pain & $0(-3 / 0)$ & $1(0 / 4)$ & 0.001 & $0(0 / 3)$ & $3(0 / 7)$ & 0.001 \\
\hline$\Delta$ NIH micturition & $1(-1 / 3)$ & $1(0 / 3)$ & 0.745 & $2(0 / 4)$ & $2(1 / 4)$ & 0.745 \\
\hline$\Delta$ NIH quality & $2(0 / 5)$ & $2(0 / 4)$ & 0.796 & $4(2 / 5)$ & $3(2 / 6)$ & 0.634 \\
\hline
\end{tabular}

\section{Discussion}

In patients undergoing PVP for BPE, the prevalence of urinary symptoms in the first postoperative month is high. Herein we demonstrated that the administration of Qurmin , an oral highly bioavailable curcumin complex, could alleviate the pain and the bothersome postoperative symptoms when compared to no treatment. Qurmin is a highly bioavailable curcumin complex that is more efficiently absorbed than the standard turmeric extract. The complexation with the naturally occurring oligosaccharide gammacyclodextrin results in a free-flowing and easily dispersible powder. The increased bioavailability seems to correlate with an enlarged surface of curcumin molecules and may explain our positive results on inflammation.

According to our RCT, Qurmin treatment is superior to no treatment in improving post-operative urinary symptoms and pain. We carefully evaluated postoperative symptoms and pain using validated questionnaires such as IPSS, NIH-CPSI and PUF. Patients in the early postoperative period may present pain and dysuria which may negatively impact on their quality of life. We recorded a 3 point improvement in IPSS after 15 days of Qurmintreatment and this can be considered a clinically significant improvement in symptoms. Nonetheless,our results confirm the efficacy of PVP in the treatment of BPH related LUTS confirming the internal validity of our results [8-15].

Curcumin is a natural phytochemical compound present in turmeric, the ground powder of the rhizomes of Curcuma longa. Curcumin has been described as having antioxidant, antiinflammatory and anti-carcinogenic properties. In vitro studies, have demonstrated specific anti-inflammatory effects of Curcumin. Grandjean-Laquerriere et al reported that curcumin attenuates the expression of ultraviolet B (UVB)-induced IL-6 and IL-8 in keratinocytes partially through inhibition of NF- $\kappa \mathrm{B}$ activation. As well, Cho et al have evaluated in an in vitro study the effect of curcumin on the expression of proinflammatory cytokines and cyclin E in TNF-alfa treated cells [16]. According to their results curcumin inhibited the expression of IL-1beta, IL- 6 and TNF but not IL-8. As well curcumin attenuated cyclin E expression. The aforementioned mechanisms provide the molecular basis to use Curcumin as an antiinflammatory molecule.

The clinical role of Curcumin has been explored in different settings. Koosiriat et al evaluated the role of Curcumin in 36 patients with chronic gastritis positive to Helicobacter Pylori. According to their results, the eradication rate of $\mathrm{H}$. pylori in patients that received OAM (Omeprazole, Amoxicillin and Metronidazole) treatment was significantly higher when compared to patients receiving curcumin (78.9\% versus 5.9\%) [17]. The levels of IL-8 mRNA expression in the OAMgroup significantly decreased after treatment, but no changes in other cytokines were found. They concluded that curcumin alone has a limited antibacterial effect and on the production of inflammatory cytokines. Madhu et al evaluated the role of Curcuma longa in a placebo-controlled trial in patients with painful knee osteoarthritis. Primary endpoint of the study was decrease in knee pain evaluated by the visual analog scale (VAS). VAS was assessed at baseline, at 21 and 42 days. According to their results improvements in pain were higher in the treatment arm when compared to placebo. They concluded that curcuma is a useful treatment option in patients with painful knee osteoarthritis [18].

In urology, very few studies have evaluated the role of curcuma to reduce inflammation with conflicting results and however none of the studies have evaluated the role of the highly bioavailable curcuma to improve symptoms after TURP $[19,20]$. In our study we confirm the role of Qurminin improving IPSS in a different population. We recorded better improvements in IPSS and in pain in patients treated with Qurmin when compared to placebo after 1 month of treatment. Moreover, no significant side effects were recorded in patients on Curcuma treatment. Most of the studies on BPH surgery evaluate surgical outcomes at 1 month and do not evaluate the immediate post-operative period. Patients may have present dysuria and storage symptoms which may negatively impact on post-operative recovery and quality of life. No specific recommendations are available for the management of patients in the early postoperative period and therefore it represents an interesting area of research. 
Citation: Cindolo L, Lombardo R, Destefanis P, Varvello F, Oriti R, et al. Efficacy of Qurmin on Post-Operative Lower Urinary Tract Symptoms After Photoselective Vaporization of the Prostate: A Multicentre Case-Control Study. J Urol Nephrol. 2021;8(1): 5.

The potential health benefits of curcumin are limited by its poor solubility, low absorption from the gut, rapid metabolism and rapid systemic elimination.It is therefore very important to use highly absorbable formulations. In a recent study, Jager et al evaluated different curcuma formulation [21]. According to their results, total curcuminoids appearance in the blood was 1.3-fold higher for a formulation with volatile oils of turmeric rhizome(CTR) and 7.9-fold higher for curcumin phytosome formulation $(\mathrm{CP})$ in comparison to unformulated standardized curcumin mixture (CS). CHC showed a 45.9-fold higher absorption over CS and significantly improved absorption over CP (5.8-fold) and CTR (34.9-fold, all p $<0.001)$.

From a chemical point of view Qurminis a cyclodextrin curcumin complex. The presence of gamma-dextrin facilitates the passage through the epithelial cells improving the curcumin concentration in blood vessels. In vitro studies have demonstrated that commercially available curcumin is very poorly bioavailable and must be dosed much higher to track its absorption. Cavacurmin reaches a concentration of $70 \mathrm{ng} / \mathrm{ml}$ after 1 hour vs $10 \mathrm{ng} / \mathrm{ml}$ for standard/ commercial curcumin. Another important pharmacodynamic property of Cavacurmin is the longer bioavailability which enables the patients to take it once daily while standard Curcumin may need several doses a day compromising the adherence to treatment. Thereafter, animal studies have observed how plasma concentration of Cavacurmin after $4 \mathrm{~h}$ reached $13 \mathrm{ug} / \mathrm{ml}$ vs $1 \mathrm{ug} / \mathrm{ml}$ for commercial products. In vitro and animal studies represent a solid base to confirm the important bioavailability of Qurmin. With this knowledge in mind we can assume that the high bioavailability of Qurmin may explain the beneficial effects of Qurmin after PVP observed by our group [22].

We have to acknowledge some limitations of our study. Although our study is well powered for the primary end point, that small number of patients may be considered a bias to prove secondary endpoints. Our study included only patients undergoing PVP and therefore the study results cannot be extended to other surgical techniques. However, a study to evaluate the role of curcuma after green light vaporization is ongoing and results will be soon available. Another possible limitation is the lack of longer follow-ups study. However, a study to evaluate the role of curcuma after Holmium enucleation of the prostate is ongoing and results will be soon available [20]. Notwithstanding all these limitations our study is the first available RCT evaluating the efficacy of Curcuma in relieving early postoperative urinary symptoms and pain.

\section{Conclusions}

According to our results the adminstration of Qurmin in patients after PVP for LUTS/PBE is more effective than no treatment in resolving the postoperative bothersome symptoms. The dietary supplement proved to be safe and very well tolerated. If further larger multicentre studies will confirm our results patients after PVP patients could systematically be treated with Qurmin.

\section{Declarations}

Ethics approval and consent to participate

All patients signed an informed consent and the internal review board approved the study.

\section{References}

1. Aydogdu O, Karakose A, Atesci YZ (2014) A clinical study comparing BIVAP saline vaporization of the prostate with bipolar TURP in patients with prostate volume 30 to $80 \mathrm{~mL}$ : Early complications, physiological changes and postoperative follow-up outcomes. J Can Urol Assoc 8: 485

2. Sipal T, Akdere $H(2020)$ The relation between the storage symptoms before and after transurethral resection of the prostate, analysis of the risk factors and the prevention of the symptoms with solifenacin. Int Braz J Urol 46: 575-584.

3. De Nunzio C, Presicce F, Tubaro A (2016) Inflammatory mediators in the development and progression of benign prostatic hyperplasia. Nat Rev Urol 13: 613-626.

4. De Nunzio C, Kramer G, Marberger M, Montironi R, Nelson W, et al. (2011) The Controversial Relationship Between Benign Prostatic Hyperplasia and Prostate Cancer: The Role of Inflammation. Eur Urol 60: 106-117.

5. De Nunzio C, Cindolo L, Gacci M, Pellegrini F, Carini M, et al. (2014) Metabolic syndrome and lower urinary tract symptoms in patients with benign prostatic enlargement: A possible link to storage symptoms. Urology 84: 1181-1187.

6. Cindolo L, Marchioni M, Emiliani E, DE Francesco P, Primiceri G, et al. (2017) Bladder neck contracture after surgery for benign prostatic obstruction. Minerva Urol Nefrol 69: 133-143.

7. De Nunzio C, Brassetti A, Gacci M, Finazzi Agrò E, Carini M, et al. (2015) Patients With Prostatic Inflammation Undergoing Transurethral Prostatic Resection Have a Larger Early Improvement of Storage Symptoms. Urology 86: 359-365.

8. Reale G, Marchioni M, Greco F, De Nunzio C, Destefanis P, et al (2019) Operative profile, safety and functional outcomes after Greenlight laser prostate surgery: Results from multicenter italian cohort analysis. Eur Urol 33499-33502.

9. Cindolo L, De Nunzio C, Greco F, Destefanis P, Bergamaschi F, et al. (2018) Standard vs. anatomical 180-W GreenLight laser photoselective vaporization of the prostate: a propensity score analysis. World J Urol 36: 91-97.

10. De Nunzio C, Lombardo R, Autorino R, Cicione A, Cindolo L, et al (2013) Contemporary monopolar and bipolar transurethral resection of the prostate: Prospective assessment of complications using the Clavien system. Int Urol Nephrol 45: 951-959.

11. Presicce F, DE Nunzio C, Gacci M, Sosnowsky R, Lombardo R, et al. (2017) The influence of the medical treatment of LUTS on benign prostatic hyperplasia surgery: do we operate too late? Minerva Urol Nefrol 69: 242252.

12. De Nunzio C, Lombardo R, Gacci M, Milanesi M, Cancrini F, et al. (2015) The Diagnosis of Benign Prostatic Obstruction: Validation of the Young Academic Urologist Clinical Nomogram. Urology 86:1032-1036.

13. Giulianelli R, Gentile BC, Mirabile G, Tema G, Albanesi L, et al. (2019) Bipolar plasma enucleation of the prostate vs. open prostatectomy in large benign prostatic hyperplasia: a single centre 3-year comparison. Prostate Cancer Prostatic Dis 22: 110-116.

14. De Nunzio C, Presicce F, Lombardo R, Carter S, Vicentini C (2017) Detrusor overactivity increases bladder wall thickness in male patients: A urodynamic multicenter cohort study. Neurourol Urodyn 36: 1616-1621.

15. Leonardo C, Lombardo R, Cindolo L, Antonelli A, Greco F, et al. (2020) What is the standard surgical approach to large volume BPE? Systematic review of existing randomized clinical trials. Minerva Urol Nefrol 72: 22-29.

16. Grandjean-Laquerriere A, Gangloff SC, Le Naour R, Trentesaux C, Hornebeck W (2002) Relative contribution of nf-kb and AP-1 in the modulation by curcumin and pyrrolidine dithiocarbamate of the UVB-induced cytokine expression by keratinocytes. Cytokine 18: 168-177.

17. Koosirirat C, Linpisarn S, Changsom D, Chawansuntati K, Wipasa J (2010) Investigation of the anti-inflammatory effect of Curcuma longa in Helicobacter pylori-infected patients. Int Immunopharmacol 10: 815-818. 
Citation: Cindolo L, Lombardo R, Destefanis P, Varvello F, Oriti R, et al. Efficacy of Qurmin on Post-Operative Lower Urinary Tract Symptoms After Photoselective Vaporization of the Prostate: A Multicentre Case-Control Study. J Urol Nephrol. 2021;8(1): 5.

\section{ISSN: 2380-0585}

18. Madhu K, Chanda K, Saji MJ (2013) Safety and efficacy of Curcuma longa extract in the treatment of painful knee osteoarthritis: A randomized placebocontrolled trial. Inflammopharmacology 21: 129-136.

19. De Nunzio C, Tema G, Lombardo R, Cicione A, Nacchia A, et al. (2019) Metabolic syndrome and smoking are associated with persistence of nocturia after transurethral resection of the prostate. Neurourol Urodyn 38:1692-1699.

20. De Nunzio C, Lombardo R, Autorino R, Cicione A, Cindolo L, et al. (2013)
Contemporary monopolar and bipolar transurethral resection of the prostate: Prospective assessment of complications using the Clavien system. Int Urol Nephrol 45: 951-959.

21. Jäger R, Lowery RP, Calvanese AV, Joy JM, Purpura M (2014) Comparative absorption of curcumin formulations. Nutr $\mathrm{J}$ 13: 11.

22. CAVACURMIN ${ }^{\circledR}$ Cyclodextrines - Wacker Chemie AG n.d. 